\title{
The pole structure of the unitary, crossing symmetric low energy $\pi \pi$ scattering amplitudes
}

\author{
Z. Y. Zhou, G. Y. Qin ${ }^{1}$, P. Zhang, Z. G. Xiao and H. Q. Zheng \\ Department of Physics, Peking University, Beijing 100871, P. R. China \\ and \\ N. Wu \\ Institute for High Energy Physics, Chinese Academy of Science, Beijing \\ 100039, P. R. China
}

November 9, 2018

\begin{abstract}
The pole structure of the low energy $\pi \pi$ scattering amplitudes is studied using a proper chiral unitarization method combined with crossing symmetry and the low energy phase shift data. It is found that the $\sigma$ pole position is at $M_{\sigma}=470 \pm 50 \mathrm{MeV}, \Gamma_{\sigma}=570 \pm 50 \mathrm{MeV}$. The existence of the virtual state pole in the $\mathrm{IJ}=20$ channel is reconfirmed. Various threshold parameters are estimated and are found in general in good agreement with the results obtained from the Roy equation analyses.
\end{abstract}

Key words: $\pi \pi$ scattering; unitarity; dispersion relations, $\sigma$ resonance PACS number: 14.40.Ev, 13.85.Dz, 11.55.Bq, 11.30.Rd

The $\sigma$ or the $f_{0}(600)$ resonance has attracted much interest in recent years [1] and more and more physicists, if not all, believe that it truly exists as a light and broad resonance. [2] There were two main difficulties to accept the $\sigma$ resonance, one is that being light and broad makes the $\sigma$ 's contribution to the phase shift hard to be distinguishable from background contributions while the latter are often out of control in various phenomenological studies. However, a careful analysis revealed that the cut contribution to the phase shift is negative in the $\mathrm{I}, \mathrm{J}=0,0$ channel $\pi \pi$ scattering [3], and thus the $\sigma$ resonance has to exist to saturate the experimental data. Another objection is that the $\pi \pi$ phase shift in the $\mathrm{I}=0, \mathrm{~J}=0$ channel does not pass $\pi / 2$ at moderately low energies which should have appeared if there exist a light $\sigma$ as required by the standard Breit-Wigner description of resonances. However it is pointed out that a light and broad resonance rejects a simple Breit-Wigner description. As

\footnotetext{
${ }^{1}$ Present address: Department of Physics, McGill University, 3600 University Street, Montreal, Quebec, H3A 2T8, Canada.
} 
a consequence a light and broad resonance's contribution to the phase shift may pass $90^{\circ}$ only at energies well above $1 \mathrm{GeV}$. 4]

Although the existence of the $\sigma$ resonance seems to be firmly established, a precise determination to the location of this resonance on the complex $s$ plane can still be challenging. A summary of the previous determinations on the pole location of the $\sigma$ resonance may be found in Ref. [5] and the results are found to be rather diverse, which is reflected in the Review of Particle Properties as the mass of the $\sigma$ ranges from $400 \mathrm{MeV}$ to $1200 \mathrm{MeV}$. In more recent studies, the pole mass of the $\sigma$ resonance is estimated to be $M \sim 500 \mathrm{MeV}$ and $\Gamma \sim 640 \mathrm{MeV}$ in Ref. [3], in Ref. [6] an extensive analysis using Roy equations indicates $M=470 \pm 30 \mathrm{MeV}, \Gamma=590 \pm 40 \mathrm{MeV}$. On the other side, the E791 experimental group claim from the $D^{+} \rightarrow \pi^{+} \pi^{-} \pi^{+}$data analysis that $M=478_{-23}^{+24} \pm 17 \mathrm{MeV}$, $\Gamma=324_{-40}^{+42} \pm 21 \mathrm{MeV}^{2}$ [7] whereas the BES collaboration has found from BES-II $J / \psi \rightarrow \omega \pi \pi$ data the $\sigma$ pole mass to be $M=541 \pm 20 \pm 32 \mathrm{MeV}$, $\Gamma=504 \pm 50 \pm 60 \mathrm{MeV}$ [8] and BES-I $J / \psi \rightarrow \omega \pi \pi$ data the pole mass as $M=434 \pm 78 \mathrm{MeV}, \Gamma=404 \pm 86 \mathrm{MeV}$. 9] Under this situation it is worthwhile to make further efforts towards a more accurate determination to the $\sigma$ pole. What we will adopt here is a new approach recently proposed by our group which is elaborated in details in Ref. [10] discussing $\pi K$ scatterings. The approach respects all known fundamental properties of $S$ matrix theory except that crossing symmetry is not implemented automatically. We will remedy the deficiency here by considering the constraints from crossing symmetry, the so called Balanchandran-Nuyts-Roskies (BNR) relations in the fit. In the following we begin by briefly review the new unitarization scheme developed in Ref. [10].

For a partial wave scattering in a given channel, the physical $S$ matrix can in general be factorized as,

$$
S^{p h y}=\prod_{i} S^{p_{i}} \cdot S^{c u t}
$$

where $S^{p_{i}}$ are the simplest $S$ matrices characterizing the isolated singularities of $S^{\text {phy }}$ [10, which are:

1. for a virtual state pole at $s=s_{0}\left(0<s_{0}<4 m_{\pi}^{2}\right)$,

$$
S^{v}(s)=\frac{1+i \rho(s) a}{1-i \rho(s) a} .
$$

The scattering length contributed by a virtual state pole defined as such is

$$
a^{v}=\sqrt{\frac{s_{0}}{4 m_{\pi}^{2}-s_{0}}} .
$$

\footnotetext{
${ }^{2}$ Corresponding to a pole mass: $M=489 \mathrm{MeV}, \Gamma=346 \mathrm{MeV}$.
} 
2. for a resonance located at $z_{0}$ (and $z_{0}^{*}$ ) on the second sheet, the $S$ matrix can be written as,

$$
S^{R}(s)=\frac{M^{2}\left[z_{0}\right]-s+i \rho(s) s \frac{\operatorname{Im}\left[z_{0}\right]}{\operatorname{Re}\left[\sqrt{z_{0}\left(z_{0}-4 m_{\pi}^{2}\right)}\right]}}{M^{2}\left[z_{0}\right]-s-i \rho(s) s \frac{\operatorname{Im}\left[z_{0}\right]}{\operatorname{Re}\left[\sqrt{z_{0}\left(z_{0}-4 m_{\pi}^{2}\right)}\right]}},
$$

where

$$
M^{2}\left[z_{0}\right]=\operatorname{Re}\left[z_{0}\right]+\operatorname{Im}\left[z_{0}\right] \frac{\operatorname{Im}\left[\sqrt{z_{0}\left(z_{0}-4 m_{\pi}^{2}\right)}\right]}{\operatorname{Re}\left[\sqrt{z_{0}\left(z_{0}-4 m_{\pi}^{2}\right)}\right]} .
$$

The expression of the scattering length contributed by a resonance defined as above is also easily obtainable. The $S^{\text {cut }}$ contains only cuts which can be parameterized in the following simple form,

$$
\begin{aligned}
S^{c u t}= & e^{2 i \rho f(s)}, \\
f(s)= & f\left(s_{0}\right)+\frac{s-s_{0}}{\pi} \int_{L} \frac{\operatorname{Im}_{L} f\left(s^{\prime}\right)}{\left(s^{\prime}-s_{0}\right)\left(s^{\prime}-s\right)} d s^{\prime} \\
& +\frac{s-s_{0}}{\pi} \int_{R} \frac{\operatorname{Im}_{R} f\left(s^{\prime}\right)}{\left(s^{\prime}-s_{0}\right)\left(s^{\prime}-s\right)} d s^{\prime},
\end{aligned}
$$

where $L=(-\infty, 0]$ and $R$ denotes cuts at higher energies other than the $2 \pi$ elastic cut. To a good approximation $R$ starts at $4 m_{K}^{2}$. The parameterization form, Eqs. (11) - (7), which may be viewed as an improved version of the Dalitz-Tuan representation, has the advantage that $S^{R_{i}}$ does not contribute to the discontinuity of $f, 10$ which means the following relation:

$$
\operatorname{Im}_{L, R} f(s)=-\frac{1}{2 \rho(s)} \log \left|S^{p h y}(s)\right| .
$$

The left cut integral in the above equation are estimated by replacing $S^{p h y}$ on the r.h.s. of Eq. (8) by $S^{\chi P T}$ which is available at two-loop order for $\pi \pi$ scatterings [11. In Fig. 11we plot the function $\operatorname{Im}_{L} f(s)$ using both tree, 1-loop and 2-loop $\chi \mathrm{PT}$ results at moderately low energies from which one may get an impression on the convergence speed of the chiral expansion. Evidences exist in the $\mathrm{I}, \mathrm{J}=2,0$ channel of $\pi \pi$ scatterings [3] and $\mathrm{I}, \mathrm{J}=\frac{3}{2}, 0$ channel of $\pi K$ scatterings [10] to support such an approximation. The present method is certainly superior to the method of Ref. 3], since now the constraint of unitarity is automatically embedded in the formalism. As a bonus of being unitary, when evaluating the left hand integral using the following equation,

$$
\operatorname{Im}_{L} f(s)=-\frac{1}{2 \rho(s)} \log \left|S^{\chi P T}(s)\right|
$$

the once-subtracted dispersion integral automatically converges owing to the logarithmic form of the integrand. This property further regulates and suppresses the bad high energy behavior of the perturbation amplitudes. Nevertheless it should be pointed out that though the dispersion integral is formally 

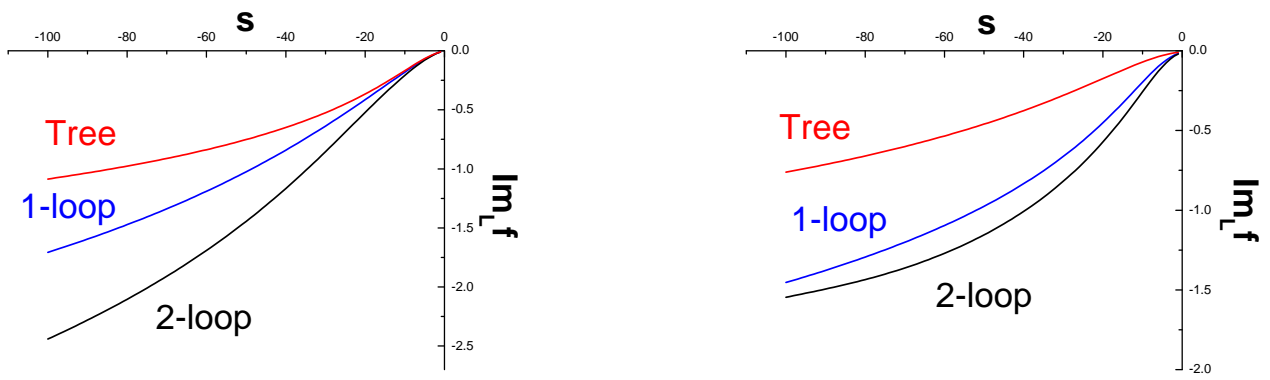

Figure 1: Tree, 1-loop and 2-loop results of $\operatorname{Im}_{L} f(s)$ from Eq. (9): left) I=0, $\mathrm{J}=0$; right) $\mathrm{I}=2, \mathrm{~J}=0$. The low energy parameters of the $O\left(p^{6}\right)$ chiral Lagrangian are taken from Ref. [12.

convergent when one uses $\chi \mathrm{PT}$ result to make numerical estimation, the contribution at high energies is beyond control theoretically due to the bad high energy behavior of $\chi \mathrm{PT}$. Therefore we introduce a cutoff parameter when evaluating the left hand integral in each channel in the numerical studies, which are determined by the minimization of $\chi^{2}$. This is of course, only a phenomenological approach. Actually, the above parametrization form can be obtained only by assuming the analyticity property on the whole cut plane, which is a consequence of Mandelstam representation. The Mandelstam representation however goes beyond what is rigorously established from field theory. Nevertheless the analyticity domain is large enough for phenomenological applications not very far from the physical region. 13.

The above discussion briefly reviewed what is already established in previous studies. However further improvement is possible. In $\pi \pi$ scatterings the kinematics is much simpler than in $\pi K$ scatterings which makes it easy to realize that the subtraction constant appeared in Eq. (II) can actually be fixed. To see this we recast Eq. (11) into the following form:

$$
\frac{1}{2 i \rho(s)} \log \left(1+2 i \rho(s) T^{p h y}(s)\right)=\frac{1}{2 i \rho(s)} \sum_{i} \log \left(S^{R_{i}}(s)\right)+f(s) .
$$

The l.h.s. of the above equation contains the normal branch points of $S$ as well as the branch points at $z_{i}$ (which denote the pole positions of $S$ ). On the r.h.s of Eq. (10) the normal branch points are presented in $f(s)$ whereas the branch points at $z_{i}$ are contained in the first term. In the absence of the long range Coulomb interaction the $\pi \pi$ scattering amplitudes $T^{p h y}$ is well behaved when $s \rightarrow 0$ and $4 m_{\pi}^{2}$. Also it is not difficult to determine the behavior of $S^{R_{i}}(s)$ when $s \rightarrow 0$ and $4 m_{\pi}^{2}$. As a consequence, one obtains, by evaluating the asymptotic behavior of each side of Eq. (10) when $s \rightarrow 0$ and $s \rightarrow 4 m_{\pi}^{2}$, the following relations:

$$
\begin{aligned}
& f(0)=0, \\
& a=\sum_{i} a^{R_{i}}+f\left(4 m_{\pi}^{2}\right)
\end{aligned}
$$

respectively. In Eq. (12) $a$ is the scattering length of the partial wave amplitude, $a^{R_{i}}$ denotes the scattering length contributed by pole $R_{i}$. One advantage 

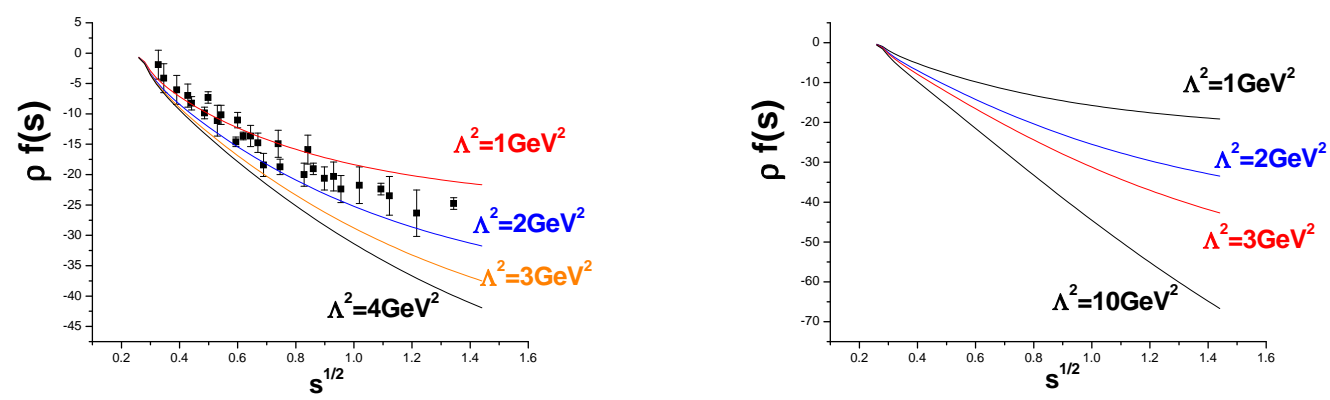

Figure 2: Different estimates on the background contribution to the phase shift. Left: in the $\mathrm{I}=2, \mathrm{~J}=0$ channel; right: in the $\mathrm{I}=0, \mathrm{~J}=0$ channel (here the right hand cut contribution is not included). The experimental data of $\delta_{0}^{2}$ are from Ref. [14.

of using Eq. (11) is that it fixes the subtraction constant appeared in Eq. (7) and suggests that the natural subtraction point in Eq. (77) is at $s_{0}=0$, that is,

$$
f(s)=\frac{s}{\pi} \int_{-\infty}^{0} \frac{\operatorname{Im}_{L} f\left(s^{\prime}\right)}{s^{\prime}\left(s^{\prime}-s\right)} d s^{\prime}+\frac{s}{\pi} \int_{4 m_{K}^{2}}^{\infty} \frac{\operatorname{Im}_{R} f\left(s^{\prime}\right)}{s^{\prime}\left(s^{\prime}-s\right)} d s^{\prime} .
$$

The validity of Eq. (131) can also be demonstrated by directly evaluating the asymptotic behavior of $f(s)$ when $s \rightarrow 0$. Starting from Eq. (13) and Eq. (8), remembering that $T^{\text {phy }}(0)$ is a constant, it is not difficult to demonstrate that the asymptotic behavior of $S^{\text {cut }}(s)$ when $s \rightarrow 0$ agrees with the l.h.s. of Eq. (10).$^{3}$ Therefore Eq. (13) is correct.

The Eq. (10) has been found very useful in Ref. [10] when studying the low lying resonance structure in $\pi K$ scatterings. Motivated by the discussion given earlier in this Letter it is worthwhile to re-examine the $\sigma$ pole in $\pi \pi$ scatterings. For such a propose, it is necessary to firstly estimate the the background contribution $f$. The left hand cut integral in Eq. (13) is estimated using two loop chiral perturbation theory results on $\operatorname{Im}_{\mathrm{L}} f$. The integral interval of the left hand integral in Eq. (13) is taken as $\left[-\Lambda_{L}^{2}, 0\right]$. In Fig. 2] we plotted the cutoff dependence of the function $f$. For the right hand cut integral in Eq. (13), we neglect its contribution in the $I J=11$ and $I J=20$ channels. In the $\mathrm{IJ}=00$ channel we will use a simple model found in the literature, [15] which gives a rather good fit to the inelasticity parameter in the energy region above $K \bar{K}$ threshold, to estimate its contribution. The integration will be truncated at some appropriately chosen cutoff scale: $\Lambda_{R}^{2} \simeq 2 \mathrm{GeV}^{2}$.

In the following we will discuss the speciality of $I J=20,11,00$ channels separately. Then we will include the constraints from crossing symmetry to make a combined fit to the data of the three channels below $1 \mathrm{GeV}$ and the so called BNR relations.

\section{$\mathrm{IJ}=\mathbf{2 0}$ channel:}

\footnotetext{
${ }^{3} \mathrm{Up}$ to a sign if the total number of bound or virtual states in a given channel is odd.
} 
According to Eq. (12), if there is no resonance or virtual state in the IJ=20 channel the scattering length will have a simple relation: $a_{0}^{2}=f\left(4 m_{\pi}^{2}\right)$, which is calculable and its value depends only on $\Lambda_{L}$ in our scheme. However such a formula gives a too large magnitude of the scattering length $a_{0}^{2}$. For example, when $\Lambda_{L}^{2}$ changes from $0.5 \mathrm{GeV}^{2}$ to $10^{6} \mathrm{GeV}^{2}, f\left(4 m_{\pi}^{2}\right)$ varies from -0.107 to -0.187 , a value too large comparing with the experimental value $a_{0}^{2}=-0.028 \pm$ 0.012 16] and the lattice results 17, though the lattice method is still not mature yet. The apparent discrepancy can be solved, recalling that there actually exists a virtual state pole in the $I J=20$ channel. 18] The pole location can be estimated from the 2 loop perturbative amplitude from $\chi \mathrm{PT}$ to be $s_{0}=0.049 m_{\pi}^{2}$ on the real axis on the second sheet of the complex $s$-plane. According to the definition of pole term adopted in the present paper, this virtual state pole's contribution to $a_{0}^{2}$ is rather large $(\simeq 0.11)$ which can not be neglected. The existence of the virtual state pole is unavoidable if chiral perturbation expansion works in the small $s$ region (actually it is a prediction of current algebra and the relativistic kinematics [18). In the present approach its existence is further tested and is found to be fully consistent with the $S$ matrix unitarization of $\chi \mathrm{PT}$. Further, the existence of such a state raises an interesting question on the deeper understanding of the physical interpretation of $S$ matrix poles and the quark hadron duality.

\section{$\mathbf{I J}=\mathbf{1 1}$ channel:}

The speciality in this channel is that the first term of the scattering length expanded in powers of pion momentum vanishes:

$$
\operatorname{Re} T_{I J}(s)=q^{2 J}\left[a_{J}^{I}+b_{J}^{I} q^{2}+O\left(q^{4}\right)\right], \quad\left(q=\frac{1}{2} \sqrt{s-4 m_{\pi}^{2}}\right),
$$

which leads to the following constraint:

$$
f\left(4 m_{\pi}^{2}\right)=-\sum_{i} a^{R_{i}}
$$

It is however not convenient to solve this constraint to reduce one resonance parameter, we therefore make the trick to subtract $f$ both at $s=0$ and at $s=4 m_{\pi}^{2}$,

$$
\begin{aligned}
f(s) & =\frac{f\left(4 m_{\pi}^{2}\right)}{4 m_{\pi}^{2}} s+\frac{s\left(s-4 m_{\pi}^{2}\right)}{\pi} \int_{L+R} \frac{\operatorname{Im} f\left(s^{\prime}\right)}{s^{\prime}\left(s^{\prime}-4 m_{\pi}^{2}\right)\left(s^{\prime}-s\right)} \\
& \rightarrow-\sum_{i} a^{R_{i}} \frac{s}{4 m_{\pi}^{2}}+\frac{s\left(s-4 m_{\pi}^{2}\right)}{\pi} \int_{L+R} \frac{\operatorname{Im} f\left(s^{\prime}\right)}{s^{\prime}\left(s^{\prime}-4 m_{\pi}^{2}\right)\left(s^{\prime}-s\right)} d s^{\prime}
\end{aligned}
$$

to meet both the threshold and the $s=0$ behavior of $f(s) .{ }^{4}$ There exists apparent disagreement between the phase shift data [19, 20] and theory in this channel, which results in a large total $\chi_{I=1, J=1}^{2}$. The contribution to the

\footnotetext{
${ }^{4} \mathrm{We}$ are in debt to I. Caprini for pointing out the mistake we made here in the previous edition.
} 
large $\chi_{11}^{2}$ comes mainly from low energy region $(\sqrt{s} \sim 0.55 \mathrm{GeV})$ and also from high energy region $(\sqrt{s} \sim 0.9 \mathrm{GeV})$, considering the extraordinarily small error bars of the data. As a consequence, the $\chi_{11}^{2}$ is very sensitive to the physical fit parameters as well as $\Lambda_{L}^{2}$ in this channel. In addition to the $\rho(770)$ pole, if we introduce another pole $\rho^{\prime}$ to simulate the effects of $\rho(1450)$ and higher resonances it will be helpful to reduce the large total $\chi_{11}^{2}$ by roughly 50 and the $\chi_{11}^{2}$ is no longer very sensitive to $\Lambda_{L}^{2}$. Also it will improve the fit value of the scattering length and the effective range parameter defined in Eq. (14). Therefore we will add a $\rho^{\prime}$ pole contribution in the fit. ${ }^{5}$ The resulting $\chi_{11}^{2}$ is still very large mainly due to the apparent disagreement between the theoretical curve and the data at low energies. Nevertheless the $\rho$ pole position is very stable against different choices of $\Lambda_{L}^{2}$. Also the $\sigma$ pole position changes very little with or without adding the $\rho^{\prime}$ pole (less than $10 \mathrm{MeV}$ ) in the combined fit as described later.

Because the consistency problem between data from Ref. [19, 20, and theory as mentioned above, it is necessary to look for experimental information from other sources. There exists accurate low energy data on pion form-factor in the $\mathrm{I}=1, \mathrm{~J}=1$ channel from the CMD-2 experiment. [21] ${ }^{6}$ However since what we need is the phase shift data, the data provided by CMD-2 experimental group are not directly usable here. The way we extract the 'experimental' data on $\delta_{1}^{1}$ is the following: we use Eq. (8) in Ref. [21] to refit the form-factor data to determine parameters, then we turn off the $\rho$ - $\omega$ mixing parameter $\delta$ and calculate the phase shift $\delta_{1}^{1}$ using again Eq. (8) of Ref. [21]. In this way we can manipulate the 'experimental' data on $\delta_{1}^{1}$. It is arbitrary to choose the center of mass energy of each data point, nevertheless we fix the center of mass energies of the 'data' set the same as the original data for form-factors provided by Ref. [21. The error bars of the phase shift 'data' are calculated using the error matrix method. In this way we obtain 43 'data' points for $\delta_{1}^{1}$.

If using our formula to fit the manipulated $\delta_{1}^{1}$ data described above we get a very small total $\chi_{11}^{2}$, which suggests that there may exist strong correlations between the error bars of the manipulated data and/or that our formula and the Eq. (8) of Ref. 21] are highly consistent. Though there appears drastic difference on total $\chi^{2}$ between the data of Ref. [19] and the data manipulated from Ref. [21, it will be shown later that the difference has only small influence on the determination of the $\sigma$ pole position.

\section{$\mathbf{I J}=\mathbf{0 0}$ channel:}

\footnotetext{
${ }^{5}$ When introducing the additional pole one has to confine it in the vicinity of $1450 \mathrm{MeV}$. Otherwise the pole will move down to $M \sim 300 \mathrm{MeV}$ with a large width to remedy the apparent disagreement between theoretical curve and data at low energies. This is unacceptable because the scattering length and the slope parameter will be totally different from the $\chi \mathrm{PT}$ results. The $\rho(1450)$ pole can not be fixed reasonably well in the present calculation since it suffers from the fact that we do not attempt to include the data around $1450 \mathrm{MeV}$, the deficiency of the data at low energies, and uncertainty of the cut-off integral when evaluating the background contribution. Therefore to introduce the $\rho^{\prime}$ pole only aims at improving the present fit results.

${ }^{6}$ We thank Prof. Leutwyler for bringing Ref. [21] to our attention.
} 
Similar to the argument given in Ref. 3], the cut contribution calculated from Eq. (13) is negative, which clearly demonstrates that the $\sigma$ resonance must exist to reproduce the observed experimental phase shift in the $\mathrm{I}=0$, $\mathrm{J}=0$ channel, which is positive and rising at low energies. The determination of the pole position of the $\sigma$ resonance is straightforward. In the $\mathrm{I}=0, \mathrm{~J}=0$ channel we begin by introducing two poles below $1 \mathrm{GeV}$ region: one is the $\sigma$ resonance under investigation and the another one corresponds to $f_{0}(980)$. With their masses and widths as fit parameters, MINUIT displays an obvious broad resonance and a narrow resonance. However, cautious readers may still ask the question whether the strong enhancement of $\delta_{0}^{0}$ below $1 \mathrm{GeV}$ is owing to the sum of residue effects of many resonances located far away from the physical region other than from a single $\sigma$ pole. To explore such a possibility we introduce a third pole without any prejudice on where it should locate. We will test whether the $\sigma$ pole location will be severely distorted by the introduction of this additional pole. The answer is no. The $\sigma$ pole moves only slightly with or without the third pole included (roughly less than $20 \mathrm{MeV}$ ). On the other side the location of the third pole is found to be very flexible when varying $\Lambda_{L}$, but always stays far away on the left. Roughly we find $M_{3}$ ranges from a few tens of $\mathrm{MeV}$ to a few hundreds of $\mathrm{MeV}$ whereas $\Gamma_{3}$ ranges from $1-3 \mathrm{GeV}$. Such a pole would better be described as pure background and its contribution to the phase shift remains small.

\section{The combined fit with constraints from crossing symmetry}

A major shortcoming of the unitarized partial wave amplitudes as described above is that crossing symmetry is not automatically built in, despite of its apparent advantages. On the other side, necessary conditions involving only low partial waves can be derived from crossing symmetry. In $\pi \pi$ scatterings, there exists crossing relations between partial wave amplitudes - BNR relations. There exists five such relations if only $s$-wave and $p$-wave are considered, i.e.,

$$
\begin{aligned}
\text { I : } & \int_{0}^{4}(s-4)(3 s-4)\left[t_{0}^{0}(s)+2 t_{0}^{2}(s)\right] d s=0 \\
\text { II : } & \int_{0}^{4}(s-4) R_{0}^{0}\left[2 t_{0}^{0}(s)-5 t_{0}^{2}(s)\right] d s=0 \\
\text { III : } & \int_{0}^{4}(s-4) R_{1}^{0}\left[2 t_{0}^{0}(s)-5 t_{0}^{2}(s)\right] d s-9 \int_{0}^{4}(s-4)^{2} R_{0}^{1} t_{1}^{1}(s)=0 \\
\text { IV : } & \int_{0}^{4}(s-4) R_{2}^{0}\left[2 t_{0}^{0}(s)-5 t_{0}^{2}(s)\right] d s+6 \int_{0}^{4}(s-4)^{2} R_{1}^{1} t_{1}^{1}(s)=0 \\
\text { V : } & \int_{0}^{4}(s-4) R_{3}^{0}\left[2 t_{0}^{0}(s)-5 t_{0}^{2}(s)\right] d s-15 \int_{0}^{4}(s-4)^{2} R_{2}^{1} t_{1}^{1}(s)=0,
\end{aligned}
$$



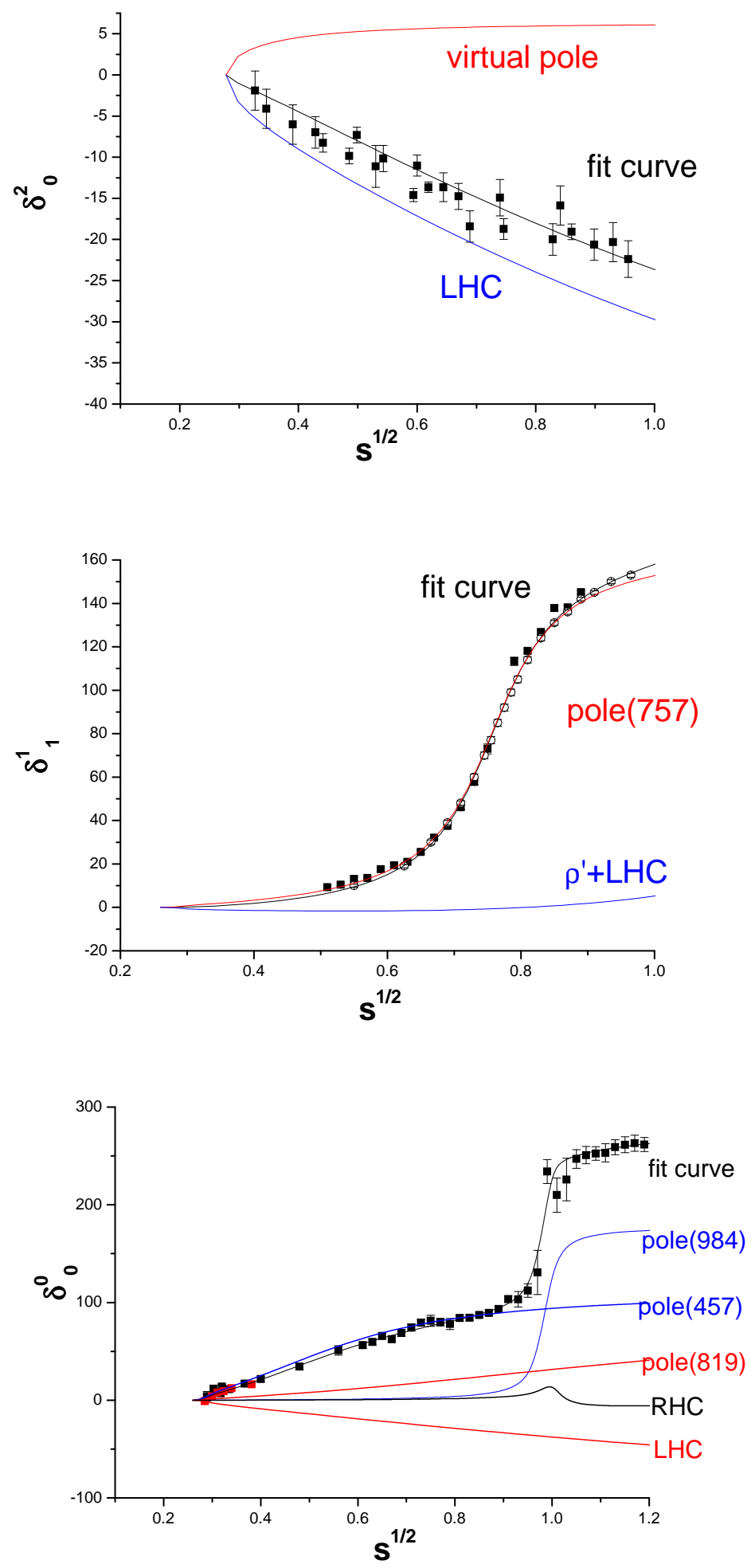

Figure 3: Fit results on phase shifts from Eq. (21). Data in the IJ=11 channel are: open circle from Ref. [19], full square from Ref. [20]. Data in the other channels are described in the text. 
where $R_{i}^{j}$ are,

$$
\begin{aligned}
& R_{0}^{0}=1, \quad R_{0}^{1}=1, \\
& R_{1}^{0}=3 s-4, \quad R_{1}^{1}=5 s-4, \\
& R_{2}^{0}=10 s^{2}-32 s+16, \quad R_{2}^{1}=21 s^{2}-48 s+16, \\
& R_{3}^{0}=35 s^{3}-180 s^{2}+240 s-64 .
\end{aligned}
$$

Dimensional parameters in Eqs. (17) [18) are expressed in unit of $m_{\pi}^{2}$ for simplicity. All these BNR relations can be rewritten in a compact form:

$$
\int_{0}^{4} d s\left[P_{00}^{i}(s) T_{0}^{0}(s)+P_{11}^{i}(s) T_{1}^{1}(s)+P_{20}^{i}(s) T_{0}^{2}(s)\right]=0,
$$

where $i$ ranges from $\mathrm{I}$ to $\mathrm{V}$ and $P_{I J}^{i}(s)$ are polynomials. We will make a combined fit on the $I J=20,11,00$ channels together with the 5 BNR relations using the penalty function method. 222] We define the penalty function as follows:

$$
\begin{aligned}
\chi_{B N R}^{2} & \equiv \frac{1}{\epsilon^{2}} \sum_{i=I}^{V}\left(E r r^{(i)}\right)^{2} \\
& \equiv \frac{1}{\epsilon^{2}} \sum_{i=I}^{V} \frac{\left|\int_{0}^{4} d s\left[P_{00}^{i}(s) T_{0}^{0}(s)+P_{11}^{i}(s) T_{1}^{1}(s)+P_{20}^{i}(s) T_{0}^{2}(s)\right]\right|^{2}}{\left[\int_{0}^{4} d s\left(\left|P_{00}^{i}(s) T_{0}^{0}(s)\right|+\left|P_{11}^{i}(s) T_{1}^{1}(s)\right|+\left|P_{20}^{i}(s) T_{0}^{2}(s)\right|\right)\right]^{2}},
\end{aligned}
$$

and define the total $\chi^{2}$ as,

$$
\chi_{t o t}^{2}=\chi_{20}^{2}+\chi_{11}^{2}+\chi_{00}^{2}+\chi_{B N R}^{2} .
$$

Altogether we have 10 physical parameters corresponding to 5 poles (3 in the $\mathrm{IJ}=00$ channel and 2 in the $\mathrm{IJ}=11$ channel). In addition we have three cutoff parameters when evaluating the background contributions in three channels. The penalty factor, $\epsilon^{-2}$, varies between $10^{-4}$ to $10^{-5}$. The rule to choose such a range is that we require that the violation of each BNR relation (corresponding to the value of $\operatorname{Err}^{(i)}$ defined in Eq. (19)) is at the level of one percent.

In the following combined fit we will test different combination of data. Firstly we use the data of $I J=11$ channel from Ref. [19, 20]. Below $1 \mathrm{GeV}$ there are 23 data points in the $\mathrm{IJ}=20$ channel [14, 39 data points in the $\mathrm{IJ}=11$ channel [19, 20] and 36 data points [14, 23, 24] in the IJ=00 channel. In the fit it is found that the total $\chi^{2}$ is sensitive to $\left(\Lambda_{L}^{2}\right)_{I J=20}$ and the best choice corresponds to $\left(\Lambda_{L}^{2}\right)_{I J=20} \simeq 3.4 \mathrm{GeV}^{2}$. On the other side the total $\chi^{2}$ is no longer sensitive to $\left(\Lambda_{L}^{2}\right)_{I J=11}$ when the $\rho^{\prime}$ pole is included. Also the total $\chi^{2}$ and all major physical outputs are insensitive to $\left(\Lambda_{L}^{2}\right)_{I J=00}$. Part of the reason behind such a phenomenon is because in the $\mathrm{IJ}=00$ channel the third pole's location is sensitive to the choice of the cutoff parameter and it absorbs the cutoff dependence of the background integral. It is found that the $\sigma$ pole location is 


\begin{tabular}{|c|c|c|c|c|c|}
\hline & Our results & $\chi \mathrm{PT}[25]$ & Roy Eqs. [6] & Exp. [16] & Unit \\
\hline$a_{0}^{0}$ & $0.211 \pm 0.011$ & $0.220 \pm 0.005$ & $0.220 \pm 0.005$ & $0.26 \pm 0.05$ & \\
$b_{0}^{0}$ & $0.264 \pm 0.015$ & $0.280 \pm 0.011$ & $0.276 \pm 0.006$ & $0.25 \pm 0.03$ & $m_{\pi}^{-2}$ \\
\hline$a_{0}^{2}$ & $-0.440 \pm 0.011$ & $-0.423 \pm 0.010$ & $-0.444 \pm 0.010$ & $-0.28 \pm 0.12$ & $10^{-1}$ \\
$b_{0}^{2}$ & $-0.785 \pm 0.010$ & $-0.762 \pm 0.021$ & $-0.803 \pm 0.012$ & $-0.82 \pm 0.08$ & $10^{-1} m_{\pi}^{-2}$ \\
\hline$a_{1}^{1}$ & $0.367 \pm 0.003$ & $0.380 \pm 0.021$ & $0.379 \pm 0.005$ & $0.38 \pm 0.02$ & $10^{-1} m_{\pi}^{-2}$ \\
$b_{1}^{1}$ & $0.563 \pm 0.003$ & $0.58 \pm 0.12$ & $0.567 \pm 0.013$ & & $10^{-2} m_{\pi}^{-4}$ \\
\hline
\end{tabular}

Table 1: Threshold parameters comparing with other works. Phase shift data in the IJ=11 channel are from Ref. [19, 20]. More accurate experimental results of $a_{0}^{0}$ and $a_{0}^{2}$ of most recent $K_{e 4}$ experiment by E865 Collaboration can be found in reference [24] : $a_{0}^{0}=0.216 \pm 0.013$ (stat.) \pm 0.004 (syst.) \pm 0.002 (theor.), $a_{0}^{2}=-0.0454 \pm 0.0031$ (stat.) \pm 0.0010 (syst.) \pm 0.0008 (theor.). See also the first reference of [28] for a different analysis of the same data.

almost inert against the variation of the cutoff parameter. Though the third pole's position is very flexible, it always wanders on the far left side of the complex $s$ plane. Taking the penalty factor $\epsilon^{-2}=10^{-4}$ is enough to constrain the violation of BNR relations at the level of 1 percent, Further decreasing it down to $10^{-5}$ does not help much in improving those BNR relations but it causes further problems on the stability of the global fit, though the center value of the $\sigma$ pole position changes very little. In below we list the fit results corresponding to (almost) the minimal $\chi^{2}$ we can find:

$$
\begin{aligned}
& \chi_{t o t}^{2}=\chi_{00}^{2}+\chi_{11}^{2}+\chi_{20}^{2}+\chi_{B N R}^{2}=29.7+214.9+41.6+4.4 \\
& M_{\rho}=757.0 \pm 0.4 \mathrm{MeV}, \quad \Gamma_{\rho}=152.2 \pm 0.6 \mathrm{MeV} \\
& M_{f^{0}}=984.5 \pm 2.3 \mathrm{MeV}, \quad \Gamma_{f^{0}}=34.4 \pm 6.8 \mathrm{MeV} \\
& M_{\sigma}=457 \pm 15 \mathrm{MeV}, \quad \Gamma_{\sigma}=551 \pm 28 \mathrm{MeV}
\end{aligned}
$$

The third pole location in the $\mathrm{IJ}=00$ channel is $M_{3}=819 \mathrm{MeV}, \Gamma_{3}=1846 \mathrm{MeV}$ with large error bars. The fit results are plotted in Fig. 3. The obtained scattering lengths and the effective range parameters are listed in table 1 where we also list several results found in the literature for comparison. From table 1 we find that our results are in general in good agreement with the $\chi \mathrm{PT}$ results [25] and especially the Roy equation analysis [6]. However, since we used the flawed low energy data in the $\mathrm{IJ}=11$ channel, ${ }^{7}$ we will also test in the following the manipulated data of $\delta_{1}^{1}$ from CMD-2. Nevertheless the problem of the data in the $I J=11$ channel does not seem to propagate into $I J=00$ and 20 channels in our approach, since the constraints from the five BNR relations on the $I J=11$ channel are rather weak because the magnitude of $T_{11}$ is suppressed in the region governed by the BNR relations.

In the second approach we use the manipulated data in the IJ=11 channel

\footnotetext{
7 if we do not include the so called $\rho^{\prime}$ pole the $b_{1}^{1}$ parameter does not agree well with the Roy equation analysis.
} 
from Ref. [21]. The solution is the following:

$$
\begin{aligned}
& \chi_{t o t}^{2}=\chi_{00}^{2}+\chi_{11}^{2}+\chi_{20}^{2}+\chi_{B N R}^{2}=29.8+0.02+41.6+5.9 ; \\
& M_{\rho}=763.0 \pm 0.2 \mathrm{MeV}, \quad \Gamma_{\rho}=139.0 \pm 0.5 \mathrm{MeV}, \\
& M_{f^{0}}=984.4 \pm 2.7 \mathrm{MeV}, \quad \Gamma_{f^{0}}=34.6 \pm 7.9 \mathrm{MeV} \\
& M_{\sigma}=459 \pm 3 \mathrm{MeV}, \quad \Gamma_{\sigma}=551 \pm 23 \mathrm{MeV} .
\end{aligned}
$$

The obtained scattering lengths and the effective range parameters in the $\mathrm{IJ}=00$ and 20 channels are found to be in satisfactory agreement with those listed in table 1. However in the $\mathrm{I}, \mathrm{J}=1,1$ channel we find that $a_{1}^{1}=3.54 \times 10^{-2}$, $b_{1}^{1}=4.27 \times 10^{-3}$, which does not seem to be compatible with the results obtained from the Wanders sum rule given in Ref. [26]. Nevertheless we find, by comparing Eq. (21) with Eq. (22), that the $\sigma$ pole location changes very little.

Finally we briefly comment on the use of another group of $\mathrm{IJ}=20$ data by Hoogland et al.. 27] The quality of solution B of Ref. 27] is not good as it gives a very large $\chi^{2}$. For solution $\mathrm{A}$, in the combined fit the central value of $a_{0}^{2}$ is $-4.09 \times 10^{-2}$. The other outputs are compatible to previous results and the fit results in a satisfactory $\chi_{B N R}^{2}$ and $\chi_{20}^{2}$. On the other side, the mass and width of the $\sigma$ resonance, will both increase roughly by $30 \mathrm{MeV}$.

A careful analysis was performed to estimate the influence of various theoretical uncertainties to the determination of the $\sigma$ pole, including the penalty factor $\epsilon$, various cutoff parameters, different treatment on IJ=11 (and also $\mathrm{IJ}=20$ ) channel data, and whether or not including the third pole. It is found that

$$
M_{\sigma}=470 \pm 50 \mathrm{MeV}, \Gamma_{\sigma}=570 \pm 50 \mathrm{MeV},
$$

where the error bars are rather conservative. The results are found in very good agreement with the results given in Ref. [6]. Different from Ref. [6], the error bars here are mainly caused by the use of different data, rather than by analytic continuation.

To conclude, using the unitarization approach developed by our group and combining the constraints from crossing symmetry, it is found that a fit to the low energy data gives reasonable results, comparing with Roy equation analyses. [6, 28 The existence of the virtual state pole in the $\mathrm{IJ}=20$ channel is reconfirmed. It is also found that crossing symmetry is crucial in correctly determining the $\sigma$ pole location. Actually using the present formulae, without the constraints from BNR relations the individual fit in the $\mathrm{IJ}=00$ channel would give a pole located at $M_{\sigma}=542 \mathrm{MeV}, \Gamma_{\sigma}=546 \mathrm{MeV}$. It is also found that the BNR relations provide a stronger correlations between the $\mathrm{IJ}=00$ and 20 channels and the 11 channel amplitude plays a relatively minor role in the BNR relations being used. The fit results end up in general in good agreement with the results of Ref. [6]. The estimated value of $\sigma$ pole position as quoted by the Ref. 2] is certainly unsatisfactory. It is desirable that newer estimates on the pole location begin to converge.

Acknowledgements: We would like to thank Profs. K. L. He, D. H. Zhang for helpful discussions on data fittings. Also we are grateful to Prof. Leutwyler 
for valuable comments and critical remarks. This work is supported in part by China National Natural Science Foundation under grant number 10491306.

\section{References}

[1] For the historical review, see, N. A. Tornqvist, Invited talk at YITP Workshop on Possible Existence of the sigma meson and its Implications to Hadron Physics, Kyoto, Japan, 12-14 Jun 2000, hep-ph/0008135.

[2] K. Hagiwara et al. (the Particle Data Group), Phys. Rev. D66 (2002)1.

[3] Z. G. Xiao and H. Q. Zheng, Nucl. Phys. A695 (2001)273.

[4] H. Q. Zheng, Talk given at International Symposium on Hadron Spectroscopy, Chiral Symmetry and Relativistic Description of Bound Systems, Tokyo, Japan, 24-26 Feb 2003, hep-ph/0304173.

[5] V. E. Markushin and M. P. Locher, Frascati Physi. Ser. 15(1999)229.

[6] G. Colangelo, J. Gasser and H. Leutwyler, Nucl. Phys. B603(2001)125.

[7] E. M. Aitala et al. (The E791 Collaboration), Phys. Rev. Lett. 86(2001) 770 .

[8] M. Ablikim et al. (The BESII Collaboration), hep-ex/0406038.

[9] J. Z. Bai et al., High Energy Phys. Nucl. Phys. 28(2004)215.

[10] H. Q. Zheng et al., Nucl. Phys. A733(2004)235.

[11] J. Bijnens et al., Phys. Lett. B374(1996)210; M. Knecht et al., Nucl. Phys. B457(1995)513. See also, H. W. Fearing and S. Scherer, Phys. Rev. D53(1996)315; J. Bijnens, G. Colangelo and G. Ecker, JHEP9902(1999)020.

[12] J. Bijnens, Nucl. Phys. B508(1997)263.

[13] see for example, A. Martin and F. Cheung, Analyticity Properties and Bounds of the Scattering Amplitudes, Gordon and Breach, New York 1970.

[14] G. Grayer et al., Nucl. Phys. B75 (1974) 189; W. Ochs, Ph.D. thesis, Munich Univ., 1974; W. Maenner, in Experimental Meson Spectroscopy - 1974 (Boston), Proceedings of the fourth International Conference on Meson Spectroscopy, edited by D. A. Garelick, AIP Conf. Proc. No. 21 (AIP, New York, 1974).

[15] B. S. Zou, D. V. Bugg, Phys. Rev. D48, 3948(1993).

[16] M. M. Nagels et al., Nucl. Phys. B147(1979) 189. 
[17] S. Aoki et al., Phys. Rev. D67(2003)014502; T. Yamazaki et al., Phys. Rev. D70(2004)074513; P. Hasenfratz, K. J. Juge and F. Niedermayer, JHEP0412(2004)030; X. N. Du, G. W. Meng, C. Miao and C. Liu, hep-lat/0404017.

[18] Q. Ang et al., Commun. Theor. Phys. 36(2001)563.

[19] S. D. Protopopescu et al., Phys. Rev. D7 (1973) 1279.

[20] P. Estabrooks and A. Martin, Nucl. Phys. B79(1974)301.

[21] R. R. Akhmetsin et al., Phys. Lett. B527 (2002)161; Corrections: Phys. Lett. B578 (2004) 285.

[22] See for example, Y. S. Zhu, Probability and Statistics in Experimental Physics, Academic press, Beijing, 1991 (in chinese).

[23] L. Rosselet et al., Phys. Rev. D15 (1977) 574.

[24] S. Pislak et al., The E865 Collaboration, Phys. Rev. D67(2003)072004.

[25] J. Bijnens, G. Colangelo, P. Talavera, JHEP 9805 (1998) 014.

[26] B. Ananthanarayan et al., Phys. Rept. 353(2001)207.

[27] W. Hoogland et al., Nucl. Phys. B69 (1974) 266.

[28] Several related Roy equations analyses are: S. Descotes-Genon et al., Eur. Phys. J. C24 (2002) 469; R. Kaminski, L. Lesniak and B. Loiseau, Phys. Lett. B551 (2003) 241. See also, J. R. Pelaez and F. J. Yndurain, Phys. Rev. D68 (2003) 074005 and the critical re-analysis by I. Caprini, G. Colangelo, J. Gasser and H. Leutwyler, Phys. Rev. D 68 (2003) 074006. 